\title{
SIWF-Award: besonderes Engagement für die Weiterbildung
}

\author{
Raphael Stolza, Nadja Jennib ${ }^{b}$ Monika Brodmann Maeder ${ }^{c}$ \\ a Dr. med., Vizepräsident des Schweizerischen Instituts für ärztliche Weiter- und Fortbildung SIWF \\ ${ }^{b}$ M.Sc., MPH, Wissenschaftliche Mitarbeiterin SIWF/FMH; ${ }^{c}$ PD Dr. med. et MME, Präsidentin des SIWF
}

\begin{abstract}
Hat sich ein früherer Weiterbildner exemplarisch für Ihre Weiterbildung eingesetzt? Haben Sie dank der hohen didaktischen Kompetenz einer Weiterbildnerin speziell erfreuliche Fortschritte in Bezug auf Kenntnisse und Fähigkeiten machen können? Dann nominieren Sie diese engagierten Weiterbildungsverantwortlichen für den SIWF-Award für besonderes Engagement in der Weiterbildung! Auch dieses Jahr können sowohl Einzelpersonen als auch verantwortliche Teams einer Weiterbildungsstätte nominiert werden.
\end{abstract}

\begin{abstract}
Wir freuen uns, bereits zum achten Mal die Ausschreibung für den SIWF-Award veröffentlichen zu können. Die zahlreichen Nominationen und das positive Echo haben uns bestätigt, dass eine solche Auszeichnung sinnvoll ist und ihren Zweck erfüllt. Die Verleihung ist deshalb fester Bestandteil der SIWF-Agenda geworden. Entscheidende Grundlage für eine optimale Vermittlung von Können und Wissen an die Weiterzubildenden ist das Engagement der Kaderärztinnen und -ärzte. Diese Aufgabe lässt sich nur beschränkt durch Pflichtenhefte definieren, viel wichtiger sind persönliches Engagement und Begeisterung. Die Belastungen in der medizinischen Arbeitswelt sind vielfältig, und die zeitlichen sowie finanziellen Ressourcen werden stets knapper, umso mehr sollten besonders aktive und motivierte Weiterbildnerinnen und -bildner auch Anerkennung erhalten. Das SIWF bietet aus diesem Grund Assistenzärztinnen und -ärzten die Möglichkeit, das ausserordentliche Engagement solcher Weiterbildungs-
\end{abstract}

\section{Jetzt Weiterbildungsverantwortliche nominieren!}

Der SIWF-Award gibt die Möglichkeit, besonders engagierten und kompetenten ärztlichen Weiterbildungsverantwortlichen und auch Teams eine Anerkennung auszudrücken. Hat ein ehemaliger Weiterbildner oder eine ehemalige Weiterbildnerin bei Ihnen einen bleibenden Eindruck hinterlassen? Dann nominieren Sie ihn oder sie für den SIWF-Award.

Füllen Sie auf der SIWF-Website (www.siwf.ch $\rightarrow$ SIWF-Projekte $\rightarrow$ SIWF Award) das OnlineFormular aus.

Einsendeschluss: 31. Juli 2021

Weitere Informationen finden Sie auf www.siwf.ch. Wenn Sie Fragen haben, erreichen Sie uns über info[at]siwf.ch oder unter 0315030600. verantwortlicher explizit zu würdigen, ohne aber eine Rangliste erstellen zu wollen.

\section{Nominierung durch Assistenzärztinnen und -ärzte}

Für den SIWF-Award können Personen nominiert werden, welche zurzeit in der ärztlichen Weiterbildung aktiv tätig sind. Im Fokus stehen Kaderärztinnen und -ärzte, welche sich persönlich für die Weiterbildung von angehenden Fachärzten und -ärztinnen einsetzen und besonders kompetent und initiativ bei der Weitergabe von Kenntnissen und Fertigkeiten sind. Auch dieses Jahr können verantwortliche Teams einer Weiterbildungsstätte nominiert werden. Nominationsberechtigt sind Ärztinnen und Ärzte, die sich zurzeit in der Weiterbildung zu einem Facharzttitel befinden oder vor weniger als einem Jahr den Facharzttitel erworben haben. Eine Nomination ist dann gültig, wenn sie durch zwei Personen gemeinsam erfolgt. Sie soll die persönliche Wertschätzung für die wahrgenommene Weiterbildungsqualität und für das Engagement der Weiterbildungsverantwortlichen ausdrücken. Damit aufgrund des Nominationsprozesses keine Vorteile oder Konflikte am Arbeitsplatz entstehen können, dürfen nur Weiterbildungsverantwortliche oder Teams nominiert werden, bei welchen die Nominierenden aktuell nicht mehr angestellt sind. Die Namen der nominierenden Personen werden nicht veröffentlicht und den Nominierten auch nicht mitgeteilt. Es wird keine «Rangliste» der Nominierten erstellt. 
Schweizerisches Institut fürärztliche Weiter- und Fortbildung SIWF FMH

Nussbaumstrasse 29

Postfach

CH-3000 Bern 16

Tel. 0315030600

info[at]siwf.ch

\section{Kriterien für eine gültige Nomination}

- Ärztinnen und Ärzte, die sich zurzeit in der Weiterbildung zu einem Facharzttitel befinden oder vor weniger als einem Jahr den Facharzttitel erworben haben;

- die Nomination muss gemeinsam durch zwei Personen erfolgen;

- Nominierende dürfen aktuell nicht mehr bei dem zu nominierenden Weiterbildungsverantwortlichen angestellt sein;

- die zu nominierende Person muss aktuell in der Weiterbildung tätig sein.

\section{Jetzt Nomination einreichen!}

Um jemanden zu nominieren, können Sie bis 31. Juli 2021 auf der SIWF-Website (www.siwf.ch $\rightarrow$ SIWF-Projekte $\rightarrow$ SIWF Award) das Online-Formular ausfüllen. Die SIWF-Geschäftsleitung überprüft, ob die Nominierung formell korrekt ist, und entscheidet abschliessend über die Gültigkeit der einzelnen Nominationen. Alle korrekt Nominierten erhalten als Würdigung ihres Engagements in der Weiterbildung eine Anerkennungsurkunde und ein Präsent. Sie werden (nach Rückfrage) auf der SIWF-Website aufgeführt und am MedEd-Symposium vom 29. September 2021 namentlich genannt.

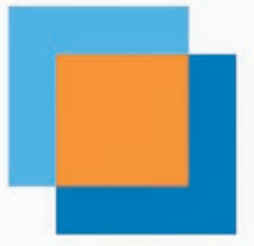

MedEd SYMPOSIUM SIWF/ISFM

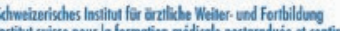

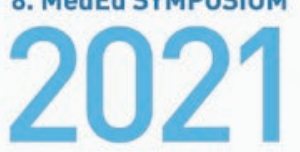

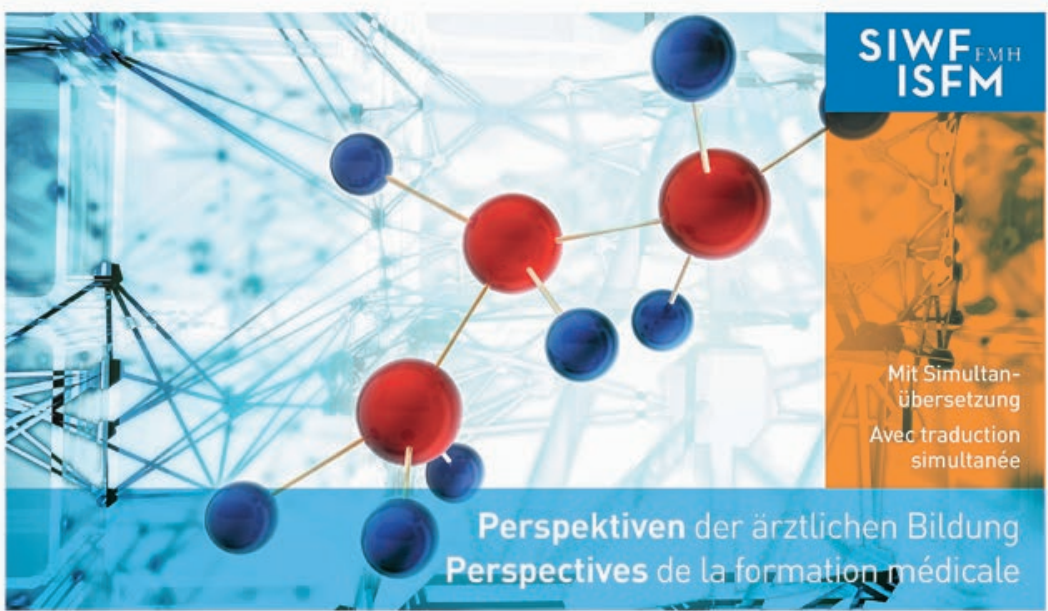

Keynote Speakers 2021

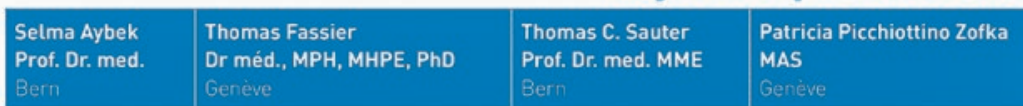

Spannende Seminare und interaktive Workshops - Séminaires passionnants et ateliers interactifs

\section{Save the Date 29. September 2021 - Casino, Bern}

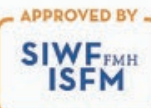
ISFM illen Fachgebieten mit 7 Credits anerkannt (SIWF-approved). e Symposium MedEd donne droit à 7 crédits dans toutes les disciplines dans le cadre de la formation continue étargie (ISFM-approved).

Anmeldung/Inscription www.congress-info.ch/meded2021 